\title{
La mobilité du capital humain : pour la productivité et le développement du Québec
}

\author{
R. Mathieu Vigneault, président et directeur général \\ Réseau Trans-Tech, Québec
}

\section{INTRODUCTION}

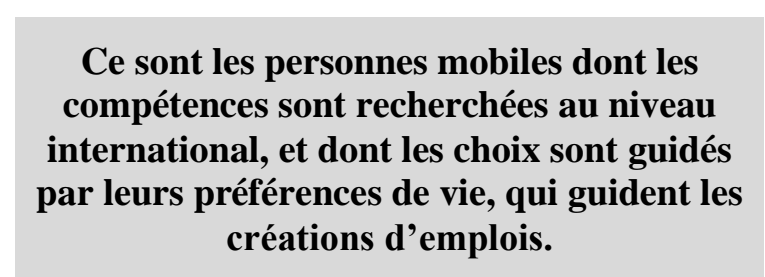

La mutation des leviers, outils et structures de soutien au développement socioéconomique des localités québécoises amène les leaders locaux à se questionner. Ainsi, ils s'interrogent sur les moyens et sur les stratégies à adopter pour conserver ou accroître l'attractivité de leur communauté auprès des investisseurs, des entrepreneurs et, de plus en plus, de la maind'œuvre qualifiée et de la population en général.

Cependant, longtemps, les municipalités ont eu pour mandat de planifier l'aménagement et l'organisation $\mathrm{du}$ quotidien d'une population plutôt homogène, déjà en place et se renouvelant de façon endogène. Aujourd'hui, nos décideurs locaux doivent de plus en plus intervenir sur la mise en valeur de leur milieu de vie et la médiation des attentes entre les différents types de citoyens, de plus en plus hétérogènes.

Nos communautés doivent faire des choix parfois difficiles qui peuvent être inspirés d'une vision à court ou à long terme, selon les intérêts des citoyens actuels ou futurs, ou bien en fonction d'une volonté de stabilité ou de développement. Cela dit, on aurait tort de penser qu'il s'agit là d'éléments nécessairement en opposition, mais ces réalités font de plus en plus appel au jugement, au doigté, à la responsabilité et au courage de nos leaders.
De là, la pérennité et le développement des territoires passent inévitablement par une plus grande attractivité de ceux-ci, un enjeu grandement affecté par la mobilité des individus, par l'individualisation des produits et services et par l'instantanéité de leur consommation, puis par la capacité à capter et à retenir l'intérêt des gens.

Dans les précédents cycles de développement, les opportunités d'emplois étaient clairement la force motrice des grandes migrations intra et inter pays. Dans le cycle de développement actuel, les emplois suivent de plus en plus les individus. En particulier, ce sont les personnes mobiles dont les compétences sont recherchées au niveau international, et dont les choix sont guidés par leurs préférences de vie, qui guident les créations d'emplois. [...] Il est évident que, alors que les villes passent d'une économie industrielle à une économie orientée sur les services à plus haute valeur ajoutée, l'importance des conditions de vie en milieu urbain augmente. [...] Les émotions complexes et variées qu'une ville peut engendrer - excitation, joie, fascination, enthousiasme, fierté, confort, peur, anxiété - sont sousjacentes à ce choix ${ }^{1}$.

Que cela nous plaise ou non, nous sommes engagés dans un monde de plus en plus compétitif. Toutefois, certains éléments positifs ressortent de cette situation, tels que la démocratisation des voies et outils de communication ainsi que le fait que les prospects sont disposés à être séduits, mais aussi de plus en plus exigeants et curieux. Bien au-delà de la capacité financière, facteur qui distingue fréquemment les plus petites localités des plus grandes, les communautés les plus dynamiques et créatives tireront avantageusement leur épingle du jeu, alors que d'autres auront d'importants défis à surmonter. 


\section{COMPRENDRE LA MOBILITÉ}

\section{La question démographique est devenue un enjeu de taille pour lequel l'État, les entreprises et les citoyens eux-mêmes constatent l'urgence d'agir.}

$\mathrm{Au}$ Québec, comme dans beaucoup d'autres juridictions de l'Organisation de coopération et de développement économiques (OCDE), la question démographique est devenue un enjeu de taille pour lequel l'État, les entreprises et les citoyens euxmêmes constatent l'urgence d'agir. Dans les faits, depuis 2015, le groupe des 15-64 ans, représentant la population active, a commencé à diminuer. Le rôle des jeunes dans la société québécoise, et particulièrement dans les régions où la population est la plus vieillissante ${ }^{\mathrm{a}}$, est donc appelé à se transformer de façon radicale.

« [Le vieillissement de la population] est un problème très important, extrêmement important au Québec, qui n'a pas encore l'attention qu'il mérite. »

- Philippe Couillard, 15 avril 2016, allocution prononcée devant la Chambre de commerce de Lévis

Concrètement, cette situation affecte un nombre grandissant d'entreprises de toutes tailles qui peinent à recruter cette main-d'œuvre si précieuse, devenue l'un des principaux facteurs de réussite et de croissance dans une économie axée sur la productivité, l'innovation et le savoir, et en marche vers l'immatériel. En même temps, plusieurs municipalités à faible croissance démographique se retrouvent aux prises avec une hausse des dépenses et des investissements, notamment liée aux besoins légitimes d'une population d'aînés en croissance.
Tant la théorie économique que la preuve empirique démontrent que la dotation en capital humain d'une nation - l'inventaire des compétences, du savoir et des attributs sociaux et personnels, dont la créativité - peut, plus que toute autre ressource, constituer des déterminants plus importants des salaires et de leur croissance, de la productivité des entreprises et de l'ensemble de la croissance et de la compétitive des entreprises et de l'ensemble de la croissance et de la compétitive économique. [...] L'investissement en capital humain ne constitue toutefois qu'une partie de l'équation; son utilisation efficace en constitue une autre partie. [...] Une forte efficacité des marchés du travail signifie que les travailleurs sont plus susceptibles d'être utilisés plus efficacement et se trouveront dans un milieu qui leur permet de donner le meilleur d'eux-mêmes².

Déjà, dans certaines communautés ou régions du Québec, ces situations contribuent à fragiliser la stabilité économique, la cohésion sociale et l'équité intergénérationnelle, favorisant une diminution de l'attractivité des territoires en question et la confirmation de spirales de dévitalisation.

Reconnue par un nombre croissant d'auteurs, de spécialistes et d'intervenants, la mobilité des individus, particulièrement celle des jeunes adultes disposant de savoir-être et de savoir-faire spécifiques, est en voie de devenir, avec d'autres approches telles que la formation de la main-d'œuvre ou l'économie sociale, un levier indispensable à la pleine participation des jeunes à la construction d'un Québec moderne et dynamique, de même qu'à la croissance économique des PME et à la pérennité de nos communautés.

\section{LA MOBILITÉ POUR L'ÉPANOUISSEMENT DU JEUNE}

Le départ des jeunes de leur région natale a longtemps été considéré comme un fléau qu'il fallait absolument endiguer. Aujourd'hui, le phénomène de la migration des jeunes est davantage compris comme un phénomène naturel - et même positif qu'il faut toutefois orchestrer et promouvoir dans une optique de développement de la personne d'abord, puis d'occupation des territoires et de vitalité socioéconomique.
Toutefois, selon les données de l'Institut de la statistique du Québec (ISQ), globalement, les Québécois sont de moins en moins mobiles ${ }^{\mathrm{b}}$. Nécessairement, ce phénomène se fait sentir dans la cohorte des 20-40 ans, soit le groupe d'individus les plus susceptibles de bouger dans les premières phases de leur vie active. Par exemple, depuis la fin des années 1990, le taux de mobilité des jeunes de la cohorte des 20-30 ans a chuté d'environ 1,5\%, 
passant d'un sommet autour de 7,2\% de cette population à plus ou moins $5,7 \%$ en 2013-2014 3 .

Alors que, pour certains, il s'agit là d'un constat plutôt positif, il s'avère qu'en se sédentarisant, les jeunes adultes québécois se détournent d'importantes occasions d'épanouissement personnel que leur offre une société vieillissante en matière d'opportunité professionnelle et d'engagement collectif/ communautaire sur l'ensemble du territoire québécois. Dans un contexte où le poids démographique et la performance économique du Québec tendent à s'effriter, le plein épanouissement de chaque membre de la collectivité devient un enjeu sociétal.

Cependant, pour remettre les choses en perspective, nous nous devons de considérer le jeune adulte dans sa globalité et de tendre l'oreille pour entendre ce qu'il nous dit, c'est-à-dire qu'il est bien davantage qu'un homo œconomicus. Bien différents de la génération des baby-boomers et de la génération $\mathrm{X}$, les jeunes de la génération $\mathrm{Y}$ (nommés aussi la génération du millénaire) portent des valeurs et des idéaux qui accordent moins d'importance à la sacrosainte réussite professionnelle, sans pour autant l'éliminer. Des concepts naguère ésotériques comme l'équilibre entre la vie professionnelle et la vie personnelle ainsi que la protection de l'environnement sont maintenant érigés en dogme.

D'autres priorités, par exemple, l'accès à la nature et à des produits locaux ou les possibilités d'engagement communautaire, constituent aujourd'hui des critères dans le choix d'une carrière ou d'un lieu de travail ou de résidence. Comme c'est de plus en plus le cas dans l'espace loisir ou l'espace relation interpersonnelle, on assiste à la reconfiguration des réseaux d'individus sur une base d'intérêts spécifiques (communauté d'intérêt) plutôt que géographique (communauté locale). Étant convaincus que le bonheur est un droit et non une conséquence, ces jeunes s'attendent à ce que leur milieu de vie reflète leur vision du monde et ils sont prêts à $\mathrm{y}$ contribuer afin de le modeler en ce sens.

$$
\begin{gathered}
\text { Dans un contexte où le poids démographique } \\
\text { et la performance économique du Québec } \\
\text { tendent à s'effriter, le plein épanouissement } \\
\text { de chaque membre de la collectivité devient } \\
\text { un enjeu sociétal. }
\end{gathered}
$$

L'enquête du Groupe de recherche sur la migration des jeunes mentionne que, parmi ceux qui font le choix de s'établir en région, les principales raisons évoquées sont la recherche d'une bonne qualité de vie $(82 \%)$ et les perspectives d'emploi (71\%). S'ajoute à cela la motivation liée à la possibilité d'acquérir une propriété $(45 \%)^{4}$.

Conséquemment, de moins en moins sensibles au concept de frontière, dans un espace de plus en plus mondialisé, plusieurs pourraient ou sont tentés d'aller s'établir dans une autre communauté correspondant davantage à leurs attentes et à leur projet de vie. D'autres, cependant, parfois ignorants de la diversité des milieux de vie ou simplement mal informés, subissent un environnement qui ne reflète pas leurs valeurs et ont du mal à mettre en valeur leur potentiel.

Plus prosaïquement, du point de vue des finances personnelles, il s'agit là d'une situation des plus préoccupantes, car, au-delà de la concordance des valeurs avec le milieu de vie, les jeunes adultes d'aujourd'hui auront à supporter, d'ici quelques années, un poids fiscal imposant auquel s'ajouteront d'autres enjeux d'ordres social, culturel, environnemental, etc.

Or, plusieurs études démontrent que c'est ce segment de la population qui est le plus vulnérable lorsque surviennent des récessions ou des soubresauts dans l'économie ${ }^{5}$. Dans les faits, le taux de chômage des jeunes est actuellement 2,3 fois plus élevé au Québec que la moyenne canadienne, soit l'écart le plus prononcé des 30 dernières années. De même, leur taux de sous-emploi au Canada atteint $27 \%$, alors qu'il se situe à $21 \%$ pour l'ensemble de l'OCDE et à $19 \%$ aux États-Unis ${ }^{6}$. Il s'agit là d'une situation qui va s'accentuer, notamment chez les jeunes, du fait de la diminution de ce groupe d'âge en proportion et de l'appel au prolongement de la période active des travailleurs expérimentés.

Parallèlement, la polarisation de $70 \%$ des jeunes adultes dans les grandes régions métropolitaines de recensement (RMR) du Québec contribue à alimenter les phénomènes du chômage et du sousemploi chez les jeunes qualifiés, et ce, particulièrement chez les Québécois issus de l'immigration. À ce sujet, de nombreuses études 
s'entendent sur les effets à moyen et à long terme sur le salaire du jeune, comme sur le PIB de l'État, d'un épisode de chômage ou de sous-emploi en début de carrière, que l'on nomme " effet scarring $»^{7}$. D'ailleurs, une étude de la Banque TD révèle que le coût économique de la chute récente de l'emploi des jeunes a eu des répercussions sérieuses sur l'économie du Canada. Le coût immédiat des pertes salariales dues au chômage est évalué à $10,7 \mathrm{G} \$$, jumelé aux coûts à long terme sur une période de 18 ans estimés à $12,4 \mathrm{G} \$$, soit au total environ $23 \mathrm{G} \$$ ou $1,5 \%$ du PIB canadien ${ }^{8}$.

Ainsi, sans faire d'association indue, mentionnons seulement que, pour le jeune qualifié, la sélection d'un milieu de vie représente un choix stratégique comportant plusieurs volets. Au-delà des questions élémentaires touchant l'adéquation valeurs/milieu de vie et occasions d'emploi, il apparaît que la mobilité professionnelle peut s'avérer plutôt rentable. En effet, selon une étude du Fonds monétaire international ${ }^{9}$ réalisée auprès de 65 pays, l'effet de la mobilité peut représenter jusqu'à $14 \%$ d'augmentation du PIB par travailleur, une donnée qui se reflète généralement sur le salaire de l'individu et sur la performance économique des collectivités.

Pris dans une perspective de mobilité aboutissant à un établissement relativement durable du jeune qualifié, le revenu s'additionne au volet du coût de la vie dans la communauté d'accueil pour déterminer le pouvoir d'achat. Ainsi, s'il n'existe pas d'absolu quant aux économies que peuvent réaliser les ménages qui s'établissent en région, plusieurs enquêtes ont tout de même démontré les avantages qui y sont rattachés, notamment sur le plan de l'accès aux coûts des services publics (loisirs, sports, culture, santé, etc.), du coût des assurances ou de l'immobilier. Concrètement, notons que la proportion des ménages qui doivent dépenser plus de $30 \%$ du revenu familial pour le poste budgétaire immobilier oscille entre $15,8 \%$ et $17,9 \%$ en milieu rural, comparativement à $24,6 \%$ en milieu urbain ${ }^{10}$.

En somme, rappelons que l'objectif n'est pas de convaincre qui que ce soit de s'établir où que ce soit, mais bien de faire valoir la mobilité comme un puissant levier de développement personnel. De notre point de vue, plus que jamais, le Québec doit s'assurer que ses jeunes adultes fassent des choix éclairés en matière d'établissement afin qu'ils puissent être autonomes et qu'ils puissent poursuivre la chaîne de solidarité intergénérationnelle.

Ainsi, pour la jeunesse québécoise, par opposition à la situation vécue par une certaine jeunesse européenne, il s'agit d'une question d'occasions à cerner et à saisir. Heureusement, les jeunes qui arrivent à l'âge adulte et qui se sont investis dans l'apprentissage d'un métier sont, par définition, à la recherche d'un environnement où mettre à profit leurs connaissances et leur capacité d'innovation. Il semble toutefois qu'ils ne sont pas prêts à faire l'économie d'un certain style de vie et qu'ils cherchent à s'établir dans un milieu qui répond spécifiquement à leurs attentes ou qui promeut des valeurs auxquelles ils s'identifient.

\section{LA MOBILITÉ POUR LA PÉRENNITÉ DE NOS COMMUNAUTÉS}

Après le défi de l'épanouissement personnel des jeunes Québécois, il convient maintenant de jeter un cil sur celui de la situation de nos communautés locales. En dépit de la diminution de la mobilité des Québécois, la métropole du Québec attire toujours autant de jeunes en provenance des régions, mais elle en perd moins au profit de ses banlieues. Cela suggère que l'importante diminution du taux de mobilité des jeunes adultes reflète davantage la capacité de la métropole à retenir sa population qu'une diminution significative de la mobilité des jeunes en provenance des milieux ruraux ${ }^{\mathrm{c}}$.
En 2013-2014, Montréal accuse un déficit total de 14700 personnes vis-à-vis de ces quatre régions limitrophes, comparativement à près de $25000 \mathrm{au}$ cours des années 2003-2004 à 2006-2007. Les échanges avec les autres régions n'ont engendré que de faibles gains ou pertes au cours de la dernière année ${ }^{11}$.

De fait, parmi les municipalités régionales de comté (MRC) situées à l'extérieur de la RMR de Montréal, 64 enregistrent des déficits démographiques chez les 15-34 ans. Selon des données établies à partir de compilations spéciales 
fournies par l'ISQ, ce sont $18 \mathrm{MRC}$ de plus qui sont en situation de fragilité.

Ainsi, d'un côté, nous constatons que la métropole attire et retient un certain capital humain, quoique la «locomotive économique du Québec » a de la difficulté à jouer pleinement son rôle ${ }^{12}$ et peine à absorber l'offre de main-d'œuvre présente sur son territoire. De l'autre, nous observons des dizaines de communautés qui ne demandent pas mieux que d'accueillir de nouvelles populations pour assurer leur pérennité et la viabilité de l'environnement socioéconomique de leurs citoyens.

Concrètement, la fiscalité locale, intimement liée à la valeur foncière du parc immobilier, est certainement l'un des volets les plus directement touchés par le phénomène du vieillissement des populations locales. En effet, pour plusieurs baby-boomers, le fait d'investir dans la construction et l'amélioration d'une résidence unifamiliale a longtemps été jugé comme un placement rentable dans une stratégie plus ou moins planifiée de préparation à la retraite. À cela s'ajoute le fait que le niveau de revenu diminue considérablement lors de la retraite, tandis que les coûts de loyer augmentent considérablement si l'on est locataire. La propriété libre d'hypothèque contribue donc à la sécurité financière de plusieurs de nos aînés. À long terme, au moment de la revente, la valeur de la propriété définira la marge de manœuvre de ceux-ci pour se reloger ${ }^{13}$.

Cela dit, le taux d'achat ${ }^{\mathrm{d}}$ de propriétés atteint un sommet au Québec chez les 25-39 ans, oscillant entre $4 \%$ et $8 \%{ }^{14}$. Naturellement, à l'opposé, les taux de vente explosent après 70 ans. Il s'installe alors, théoriquement, un certain équilibre, par lequel les jeunes ménages qui font l'acquisition d'une maison à l'intérieur des paramètres d'un marché équilibré contribuent directement à la sécurité financière des aînés qui s'en départissent.

Or, le marché immobilier dépend majoritairement de paramètres territoriaux tels que la démographie, le marché de l'emploi, l'aménagement du territoire, les infrastructures, etc. Conséquemment, la réalité démographique que vivent actuellement plusieurs communautés à caractère rural fragilise cet équilibre et favorise même une pression à la baisse du prix des maisons. Cela affecte considérablement déjà la sécurité financière de nos aînés et, en même temps, les revenus fiscaux de nos municipalités, basés sur la valeur des propriétés.

La fiscalité locale, intimement liée à la valeur foncière du parc immobilier, est certainement l'un des volets les plus

directement touchés par le phénomène du vieillissement des populations locales.

Ainsi, pour les jeunes familles désireuses d'acquérir une première résidence, les régions offriront de nombreuses occasions d'investissement. Cette avenue est d'autant plus intéressante pour les immigrants qui, selon une étude de la Banque Scotia, présentent une progression du taux de propriétés supérieure à celle des Canadiens d'origine .

Pour leur part, nos décideurs locaux verront, dans l'arrivée de nouveaux ménages, certes de nouveaux citoyens, bénévoles et contribuables, mais aussi des alliés informels pour assurer le bien-être de nos aînés et la stabilisation de l'assiette fiscale de la municipalité.

Au-delà du marché de l'immobilier, la mobilité des jeunes adultes qualifiés ouvre aussi la porte à des impacts significatifs, notamment dans le vaste domaine de la santé. Par exemple, les projections prévoient que la proportion de personnes de plus de 65 ans atteindra $20 \%$ en 2031 , puis $25 \%$ en 2036. Fort heureusement, la majorité des babyboomers devraient vieillir en bonne santé, ce qui leur permettrait de conserver leur autonomie et leur habitation. Cependant, une proportion significative de personnes âgées connaîtra des problèmes de santé pour lesquels une assistance est nécessaire : on parle ici du tiers des 75 ans et plus ${ }^{15}$. En 2031, cette cohorte aura augmenté de $133 \%$, soit deux fois plus qu'en Europe pour la même période.

Depuis l'adoption de la politique de soutien à domicile Chez soi : le premier choix en 2003, le ministère de la Santé et des Services sociaux (MSSS) s'intéresse de près au développement du soutien à domicile des personnes en perte 
d'autonomie. Et pour cause, car, au terme de l'année financière 2011-2012, l'État québécois avait investi 2,9 G\$ dans le soutien à l'autonomie des personnes âgées, soit $67 \%$ du budget du MSSS et plus de $1 \%$ du PIB de la province. Selon l'OCDE, ces sommes pourraient représenter de 2 à $3 \%$ du PIB en 2050.

Pour le ministère, la famille est appelée à jouer un rôle de plus en plus important, notamment à titre d'aidante naturelle pour les activités de la vie quotidienne. Sur le plan émotif, ce soutien pourra prendre la forme d'une présence réconfortante, divertissante et stimulante pour assurer le bienêtre de l'aîné. Évidemment, tous ces avantages et ces bienfaits surviendront pour peu que les personnes puissent être en présence les unes avec les autres. Dans le cas contraire, c'est l'aîné luimême qui est appelé à se déraciner pour se rapprocher des centres de services urbains, soit $10 \%$ des ménages de plus de 65 ans $^{16}$.

Si la mobilité des jeunes adultes offre d'intéressantes perspectives socioéconomiques pour les individus et les collectivités sur le plan de l'immobilier, mais aussi dans le secteur de la santé publique, elle est appelée à avoir des impacts tout aussi significatifs du côté de la sécurité. Dans les faits, le bassin de population est intimement lié à la présence régulière ou non des forces policières. Celui-ci joue aussi un rôle de premier plan quant à la masse critique de population capable de fournir un nombre suffisant de pompiers volontaires. Même constat du côté communautaire, avec la disponibilité de bénévoles favorisant l'entraide ou le maintien de l'offre de services publics (écoles et CPE).

Pour les collectivités locales, il ne fait aucun doute que l'énergie et les ressources consenties à développer l'attractivité et à favoriser l'accueil de nouvelles populations constituent un investissement hautement rentable. À titre d'exemple, une évaluation très conservatrice des retombées de l'action de Place aux jeunes en région en 20142015 s'élève à plus de 34 M\$ (36 M\$ l'année précédente) répartis dans les 58 MRC partenaires de l'initiative. L'effort combiné du gouvernement du Québec et des communautés partenaires, qui financent le projet depuis 25 ans, représente un ratio de 1/10 en retombées pour chaque dollar investi dans la promotion et l'accompagnement à la mobilité de jeunes adultes diplômés.

Toutefois, encore beaucoup de travail reste à accomplir, car, selon les indications du Conference Board du Canada (CBC), près de la moitié (41) des MRC ou territoires équivalents de la province ont un taux d'attraction ${ }^{\mathrm{f}}$ inférieur à la moyenne québécoise et près du tiers (31) présentent un score à peu près égal à la moyenne. Ainsi, l'attractivité et la capacité d'accueil des communautés représentent « un défi qui sera au cœur même de la construction de communautés durables $»^{17}$. L'attraction, l'accueil et l'enracinement de nouvelles populations demeurent des rouages importants de la prospérité future de toute communauté, qu'elle soit rurale ou urbaine.

\section{LA MOBILITÉ POUR LA CROISSANCE DE NOTRE ÉCONOMIE}

Globalement, bien que la question du vieillissement des populations soit un phénomène large qui touche l'ensemble des États membres de l'OCDE, l'importance avec laquelle il se vit au Québec justifie largement la mobilisation de l'ensemble des acteurs interpellés par la performance économique du Québec. En effet, les modifications dans la structure de l'âge des populations et dans les fluctuations quantitatives sont matière à influencer notre économie de façon appréciable, certes en ce qui a trait au niveau et au mode de consommation des gens, mais aussi et surtout à la place du capital humain dans cette économie. Ainsi, propulsée par une économie du savoir, la qualité de la maind'œuvre québécoise constitue l'un des éléments essentiels pour les entreprises, notamment dans le choix de l'emplacement de leurs installations de production et des processus commerciaux ${ }^{18}$.

Nécessairement, le premier élément d'une maind'œuvre de qualité est sa disponibilité, qui, sur un territoire aussi vaste et peu densément peuplé que le Québec ou le Canada, doit nécessairement faire appel à la mobilité. Celle-ci, qui peut être occupationnelle ou géographique, permet aux travailleurs d'aller là où l'on a le plus besoin d'eux ${ }^{19}$. Le second élément est sa contribution à la création de valeur ajoutée dans les entreprises et, par voie de conséquence, de richesse pour l'ensemble de la collectivité. 


\section{Graphique 1 - Provenance de l'offre de main-d'œuvre au cours des années 2012 à 2021}

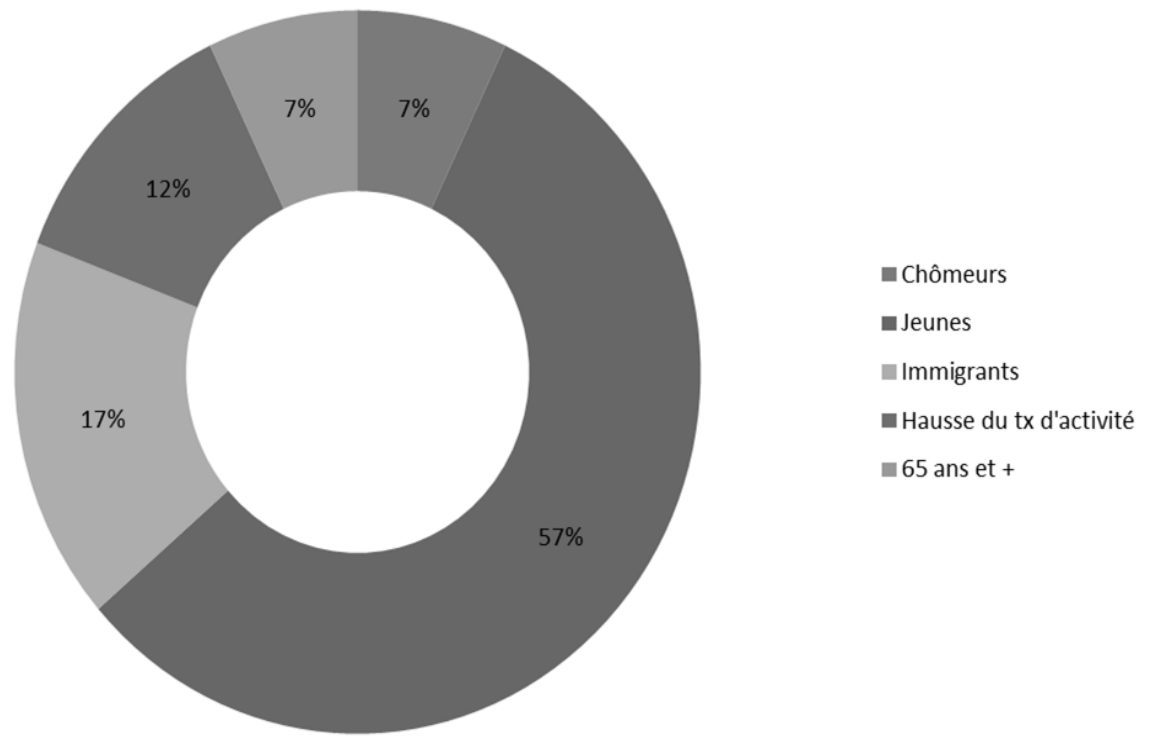

Or, les phénomènes de vieillissement des populations en milieu rural et de concentration des jeunes adultes dans les milieux urbains inquiètent. Cette situation est en voie de devenir sérieusement problématique, car c'est cette jeunesse qui doit assurer la plus grande partie des quelque 1,4 million de postes à pourvoir d'ici 2022, et ce, dans toutes les régions du Québec. Cela fait dire à plusieurs qu'une meilleure adéquation capital humain/emploi est hautement souhaitable, voire prioritaire.

Heureusement, le contexte socioéconomique du Québec et de ses régions propose de très nombreuses occasions de développement socioprofessionnel aux jeunes adultes qualifiés.

Entre 2000 et 2012, le marché de l'emploi québécois a vu le nombre d'emplois offerts dans le secteur des services augmenter de $25 \%$. Au passage, les régions ont pu capter une certaine partie de la croissance de l'emploi dans les secteurs des services à la production, à la consommation et gouvernementaux, complétant le tableau d'une économie en marche vers une domination du secteur des services.

Alors que notre système de création de la richesse collective chemine vers une économie de l'immatériel, l'ISQ annonce que la part d'emplois de qualité élevée ${ }^{\mathrm{g}}$ a doublé depuis 1997, passant de
10 à $20 \%$ pour les $15-29$ ans et de 30 à $35 \%$ chez les 30 ans et plus. Cette information est confirmée par Emploi-Québec, qui prévoit que les principaux niveaux de compétence en croissance seront, et ce, bien au-delà des autres, les niveaux technique I (DEC) et professionnel ${ }^{20}$. En d'autres termes, une part en croissance des jeunes Québécois aura accès à un emploi qualifié ou sera sollicitée pour en occuper un au cours des prochaines années, et ce, en ville comme en région.

Devant cette situation, la Fédération canadienne de l'entreprise indépendante (FCEI) soutient que, parmi les plus importantes contraintes à la croissance des petites et moyennes entreprises (PME), la pénurie de main-d'œuvre qualifiée et la pénurie de main-d'œuvre non qualifiée se classaient respectivement en $2^{\mathrm{e}}$ et $6^{\mathrm{e}}$ rang $^{21}$. Plus spécifiquement, il s'agirait du facteur qui affecte le plus négativement la productivité des fabricants québécois, selon le regroupement Manufacturiers et exportateurs du Québec (MEQ).

« Le problème qu'on a, c'est qu'il n'y a pas la rencontre entre l'emploi offert et l'employé disponible. »

- Philippe Couillard, 15 avril 2016, allocution prononcée devant la Chambre de commerce de Lévis 
À ce sujet, l'ensemble des intervenants s'accorde pour affirmer que, si aucune action stratégique n'est menée d'ici 2020, alors que bon nombre de babyboomers auront pris leur retraite, les problèmes devraient se manifester plus sérieusement ${ }^{22}$.

En second lieu, la situation problématique évoquée plus haut touche la question de l'entrepreneuriat, qui représente un défi de taille auquel font face les régions ${ }^{\mathrm{h}}$ et dont la mobilité constitue une piste de solution plus intéressante. À cet effet, les résultats de différentes enquêtes convergent, notamment en ce qui concerne les besoins de nouveaux entrepreneurs $(38000$ au cours des prochaines décennies) pour prendre la relève des baby-boomers ${ }^{23}$.

Concrètement, le tissu économique québécois est composé presque en totalité de PME, qui génèrent $50 \%$ du PIB. Elles constituent la grande majorité des créateurs d'emplois et contribuent au maintien d'environ $57 \%$ de tous les emplois de la province. Selon la firme Raymond Chabot Grant Thornton, l'incidence de la disparition de ces PME serait très importante et affecterait l'ensemble des régions du Québec, en plus de créer une pression sur les finances publiques, menaçant même la pérennité de nos programmes sociau ${ }^{24}$.

Si l'on peut voir dans cette situation un problème potentiel, on peut aussi y déceler tout le potentiel et les occasions pour des milliers d'entrepreneurs en devenir. Il reste désormais à rejoindre ces entrepreneurs de la relève pour déterminer leur profil et pour leur proposer ces occasions. À noter qu'en général les individus se lancent en affaires avant 45 ans. Par ailleurs, en tenant compte de la mutation de l'économie vers une économie de l'immatériel complexifiée et ultrarapide, les entrepreneurs de demain devront être qualifiés et bien branchés sur l'environnement externe de leur éventuelle entreprise (clients, fournisseurs, concurrence, etc.). Selon ce portrait et considérant les données de concentration de la jeunesse québécoise évoquées plus haut, un constat s'impose: une partie importante du bassin des futurs entrepreneurs se trouve en milieu urbain.

Les occasions d'affaires présentes en région recèlent donc un fort potentiel actuellement très peu exploité. Si plusieurs modèles d'affaires peuvent être mis en œuvre afin d'assurer une continuité par des acteurs de la communauté d'origine, il apparaît, comme c'est déjà le cas dans plusieurs territoires, que le transfert peut parfois ne pas se réaliser en raison du manque de capital humain sur place.

Cette affirmation est également valable pour les nouvelles occasions d'affaires, c'est-à-dire des occasions brutes, encore inexploitées. Considérant le nombre grandissant de jeunes professionnels en quête de défi et d'autonomie, et disposés à emprunter cette voie, il y a là un filon à exploiter, et ce, particulièrement du côté de la microentreprise ou du travailleur autonome.

À ce sujet, Statistique Canada révèle que les travailleurs autonomes propriétaires d'une entreprise constituée en société ont des revenus significativement supérieurs à ceux des travailleurs salariés ${ }^{25}$. Plus intéressant encore, l'étude mentionne qu'ils ont un patrimoine financier 2,7 fois plus élevé que celui des salariés $(520 \mathrm{k} \$$ contre $195 \mathrm{k} \$$ ) et qu'ils dépensent considérablement plus, notamment sur le plan de l'économie domestique (ex. : alimentation, vêtements, soins, loisirs, etc.).

Un troisième volet pour lequel la flexibilité du marché du travail est appelée à jouer un rôle de premier plan est celui de la productivité des entreprises. En effet, selon une étude de HEC Montréal ${ }^{26}$, ce lien est mis en évidence par le fait que l'on peut montrer que tout changement dans la productivité peut être décomposé en trois termes: l'intégration de meilleurs processus d'affaires ou de technologies (effet infrafirme), l'attraction chez les entreprises les plus productives de la main-d'œuvre (effet interfirmes) et le remplacement des entreprises moins productives par d'autres qui le sont davantage (effet net d'entrée). En y regardant de plus près, on constate que deux des trois effets évoqués sont directement liés à la mobilité de la main-d'œuvre, soit l'effet interfirmes et l'effet net d'entrée.

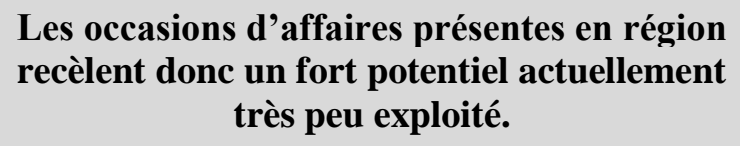


\#« Le grand problème de l'économie du Québec, c'est la productivité. »

- Philippe Couillard, 15 avril 2016, allocution prononcée devant la Chambre de commerce de Lévis

Cependant, en comparant le niveau de contribution de la mobilité de la main-d'œuvre à la croissance de la productivité des entreprises québécoises avec celui du reste du Canada et de l'Ontario (qui a une structure économique semblable à celle du Québec), on constate qu'un peu plus de $50 \%$ de l'évolution de la productivité au Canada et en Ontario est attribuable à la mobilité de la main-d'œuvre. Cette contribution n'est que de $10 \%$ dans la Belle Province. L'écart est encore plus flagrant avec certains pays d'Europe de l'Est ou avec le Danemark, où on atteint $75 \%$ de contribution ${ }^{27}$.
Cela permet d'affirmer que le niveau de flexibilité/fluidité de notre marché de l'emploi est nettement sous-optimal et qu'une plus grande mobilité du capital humain pourrait contribuer à augmenter considérablement la productivité de nos entreprises.

Dans une économie comme celle du Québec, qui doit presque essentiellement miser sur l'exportation pour créer de la richesse, l'évolution de la valeur du PIB par travailleur est un enjeu majeur pour l'État en général. À ce sujet, une autre étude, réalisée par le Centre sur la productivité et la prospérité de HEC Montréal, démontre très clairement que ce sont les régions et plus particulièrement les régions dites ressources qui ont généré la croissance de la productivité la plus importante entre 2002 et $2012^{28}$.

Tableau 1 - Croissance de la productivité du travail 2002-2012

\begin{tabular}{|l|c|c|}
\hline \multicolumn{2}{|c|}{$\begin{array}{c}\text { Croissance de la productivité du } \\
\text { travail 2002-2012 }\end{array}$} & $\begin{array}{c}\text { en \$/heure } \\
\text { travaillée }\end{array}$ \\
\hline Nord-du-Québec & $213 \%$ & $132,77 \$$ \\
\hline Côte-Nord & $34 \%$ & $96,88 \$$ \\
\hline Abitibi-Témiscamingue & $29 \%$ & $55,59 \$$ \\
\hline Saguenay-Lac-Saint-Jean & $17 \%$ & $50,55 \$$ \\
\hline Montréal & $10 \%$ & $49,42 \$$ \\
\hline Capitale-Nationale & $5 \%$ & $44,07 \$$ \\
\hline Laval & $-3 \%$ & $42,32 \$$ \\
\hline
\end{tabular}

Bien entendu, l'extraordinaire performance de nos régions ressources doit être nuancée en fonction de leur poids réel dans l'économie totale du Québec, notamment en rapport avec le nombre total d'heures travaillées dans la province, qui, luimême, est intimement lié aux secteurs économiques impliqués, au nombre d'entreprises et à la démographie. Toutefois, même si le portrait présenté dans le tableau précédent s'inverse quand on considère la contribution à l'ensemble du PIB de la province, compte tenu de l'importante masse critique de la métropole, il n'en demeure pas moins que le plus important potentiel de croissance demeure en milieu rural. Or, la grande majorité de la main-d'œuvre qualifiée la plus affectée par le sous-emploi se trouve à Montréal. 
Il est facile de conclure qu'un meilleur appariement de la main-d'œuvre entre les bassins de jeunes adultes diplômés et potentiellement mobiles et les employeurs à la recherche de ces ressources qualifiées générerait une amélioration significative de la croissance économique du Québec dans son ensemble, et ce, sans compter sur les bénéfices indirects de la diminution du sous-emploi dans les régions métropolitaines.

En somme, pour reprendre les mots du premier ministre Couillard, le vieillissement de la population est un problème extrêmement important qui n'a pas encore eu l'attention qu'il mérite. Pour faire face à ce défi structurel, plusieurs pistes de solution adressant plusieurs variables doivent être mises de l'avant, dont une plus grande mobilité des Québécois.

À la lumière de ce qui a été présenté ici, il ne fait plus aucun doute que la mobilité est un levier puissant de développement. D'abord pour les jeunes adultes, qui, sans renier un certain intérêt pour les milieux urbains, sont de plus en plus nombreux à démontrer une curiosité grandissante pour les petites villes ou la campagne. Concrètement, pour beaucoup de jeunes adultes, les milieux ruraux portent en eux des valeurs proches des leurs, telles que l'équilibre entre la vie professionnelle et la vie personnelle, la protection de l'environnement, la consommation responsable, etc. Pour peu que les communautés locales démontrent de l'ouverture, ces jeunes adultes sont tout disposés à les considérer comme un milieu de vie favorisant leur épanouissement personnel.

Il est facile de conclure qu'un meilleur appariement de la main-d'œuvre entre les bassins de jeunes adultes diplômés et potentiellement mobiles et les employeurs à la recherche de ces ressources qualifiées générerait une amélioration significative de la croissance économique du Québec.

Par ailleurs, à la fin de leur parcours scolaire, qu'ils ont majoritairement réalisé dans l'une des quatre grandes RMR du Québec, les jeunes diplômés doivent rivaliser avec d'autres travailleurs, dans un marché de l'emploi toujours plus compétitif. Afin d'éviter les épisodes de chômage ou de sous-emploi, plusieurs auront avantage à se tourner vers les régions, où ils seront davantage en mesure de trouver un emploi à la hauteur de leurs compétences. Ce faisant, ils pourront mettre rapidement leur expertise à contribution et acquérir une expérience variée, essentielle à leur accomplissement professionnel. Parallèlement à leur engagement dans le marché du travail, ces mêmes jeunes adultes pourront commencer à bâtir leur pouvoir d'achat et leur patrimoine financier, et ainsi assumer pleinement leur rôle dans la collectivité. Ils seront aidés en cela par un coût de la vie généralement moins élevé que dans les milieux urbains, particulièrement lorsque vient pour beaucoup d'entre eux le projet d'achat d'une propriété.

Les effets positifs de la mobilité des individus se font aussi sentir à l'échelle de la collectivité locale/régionale. En effet, sachant qu'une des principales sources de revenus des municipalités est l'impôt foncier, le poids du vieillissement de la population peut être lourd à porter à long terme. À l'inverse, une communauté qui réussit à se renouveler en additionnant à la croissance naturelle l'arrivée de nouveaux ménages favorisera le maintien, voire le développement de son assiette fiscale et l'apparition de nouveaux champs tarifaires. En plus de l'impact direct sur l'autonomie et sur la capacité de prise en charge de la localité, cet effet positif est aussi palpable du côté de nos aînés, qui, pour beaucoup, ont misé sur la valeur de revente de leur résidence pour s'assurer une certaine sécurité financière.

De même, en complément de l'aspect financier qui demeure central, l'arrivée de nouvelles populations permet d'entretenir, voire de développer l'engagement solidaire, un important levier de développement socioéconomique. D'abord à l'échelle de la famille, par exemple, dans l'accompagnement des aînés à domicile, le gardiennage d'enfants ou l'entraide familiale.

Puis, à l'échelle de la collectivité, par le bénévolat et l'implication sociale, par exemple, dans l'animation sportive ou culturelle, l'entretien et la promotion du patrimoine ou encore la démocratie locale. Parallèlement, la mobilité favorise le maintien d'une masse critique de citoyens justifiant le maintien ou l'investis- 
sement de services publics, comme dans le cas des écoles primaires, des CPE, de la sécurité policière ou civile et du service incendie.

Enfin, à l'échelle nationale, la mobilité du capital humain joue un rôle extrêmement important quant à la stabilité et à la croissance de notre économie. D'entrée de jeu, elle permet une optimisation de chaque ressource dans un contexte de raréfaction de la main-d'œuvre, tout en limitant le sous-emploi chez les jeunes, un phénomène collectivement très coûteux. Ainsi, elle permet aux entreprises situées aux quatre coins de la province d'avoir accès à une maind'œuvre de renouvellement qualifiée et à court terme. En cela, la mobilité intervient en complément aux efforts de formation de la maind'œuvre, qui, elle, génère ses effets concrets pour les employeurs à moyen et à long terme. Bien orchestrée, cette mobilité joue un rôle de premier plan dans la création de richesse en favorisant concrètement l'augmentation de la productivité des entreprises. Dans les faits, un marché de l'emploi flexible et fluide vient soutenir les entrepreneurs et favoriser l'investissement, l'innovation et l'exportation de nos PME et, donc, l'enrichissement collectif.

Comme si cela n'était pas encore assez, la mobilité du capital humain intervient aussi du côté de l'entrepreneuriat en favorisant tacitement, lorsque nécessaire, la relève d'entreprise par de jeunes entrepreneurs dynamiques venus d'une autre région, voire d'un autre pays. Informés des occasions de relève d'entreprise ou d'emploi qui n'ont pas encore été valorisées, ces entrepreneurs venus d'ailleurs deviennent, avec d'autres leaders de la communauté, d'importants acteurs dans l'établissement de spirales positives de développement.

\section{BIBLIOGRAPHIE}

${ }^{1}$ Moonen, T., Moiret, E. et Clark, G. (2015). Capacité d'attraction : la dynamique des villes. Dans L. BourdeauLepage et V. Gollain (dir.), Attractivité et compétitivité des territoires : théories et pratiques (p. 13). Paris, France : CNER. P.13.

${ }^{2}$ Canada 2020. (2014). Le chômage et le sous-emploi chez les jeunes : obstacles à la réalisation du plein potentiel économique du Canada. Ottawa : Gouvernement du Canada. Repéré à http://canada2020.ca/fr/ news-ideas/rapports-de-recherche/
${ }^{3}$ Institut de la statistique du Québec. (2015). La migration interrégionale au Québec en 2013-2014 : nouvelle baisse de la mobilité. Coup d'œil sociodémographique, 37, 115. Repéré à http://www.stat.gouv.qc.ca/statistiques/populationdemographie/bulletins/coupdoeil-no37.pdf ${ }^{4}$ Gauthier, M. et al. (2006). La migration des jeunes au Québec : rapport national d'un sondage 2004-2005 auprès des 20-34 ans du Québec. Québec, Québec : Observatoire Jeunes et Société, INRS. Repéré à https://www.placeauxjeunes.qc.ca/file_library/Migratio n_jeunes_Sondage_2006_GRMJ.pdf

${ }^{5}$ Christopher, R. (2014). What now? Addressing the burden of Canada's slow-growth recovery: Canadian macro policy after the crisis, Commentary No 413, Toronto, Ontario: C.D. Howe Institute. Repéré à https://www.cdhowe.org/sites/default/files/attachments/ research_papers/mixed//Commentary_413.pdf

${ }^{6}$ Canada 2020, op. cit.

${ }^{7}$ Schwerdtfeger, M. (2013). Assessing the long term cost of youth unemployment. TD Economics, Special report. Repéré à https://www.td.com/document/PDF/ economics/special/ms0113_YouthUnemp.pdf

${ }^{8}$ Ibid.

${ }^{9}$ Poirson, Helene (2000). The impact of intersectoral labor reallocation on economic growth, IMF Working Paper, WP/00/104. Washington, DC: International Monetary Fund. Repéré à https://www.imf.org/ external/pubs/ft/wp/2000/wp00104.pdf

${ }^{10}$ Société d'habitation du Québec (SHQ). (2011). Le vieillissement de la population et le logement : exploration en banlieue, Bulletin Habitation Québec, 5(2). Repéré à http://www.habitation.gouv.qc.ca/ fileadmin/internet/publications/H01152.pdf

${ }^{11}$ Institut de la statistique du Québec. (2015), op. cit.

${ }^{12}$ Arcand, A., Gagné, R., Homsy, M. et Lussier, J. (2014). Montréal : boulet ou locomotive? L'importance de la métropole pour l'économie du Québec. Montréal, Québec : Institut du Québec.

${ }^{13}$ Société d'habitation du Québec (SHQ), op. cit.

${ }^{14}$ Cortellino, F. et Hughes, K. (2012). Vieillissement de la population au Québec : se dirige-t-on vers un surplus de propriétés existantes à vendre? Dans S. Rheault, S. et J. Poirier (dir.), Le vieillissement démographique : de nombreux enjeux à déchiffrer (p. 225-231). Québec, Québec : Institut de la statistique du Québec. Repéré à http://www.stat.gouv.qc.ca/ statistiques/conditions-viesociete/vieillissement.pdf

${ }^{15}$ Ménard, F.-P. et Le Bourdais, C. (2012). Impact de la diversification des trajectoires familiales sur les échanges intergénérationnels. Dans S. Rheault et J. Poirier (dir.), Le vieillissement démographique : de nombreux enjeux à déchiffrer (p. 131-145). Québec, Québec : Institut de la statistique du Québec. Repéré à http://www.stat.gouv.qc.ca/statistiques/conditions-viesociete/vieillissement.pdf. 
${ }^{16}$ Leduc, S. (2010). Les baby-boomers et le logement. Bulletin Habitation Québec, 5(1), 16. Repéré à http://www.habitation.gouv.qc.ca/fileadmin/internet/pu blications/H01051.pdf

${ }^{17}$ Conference Board du Canada. (2009). Les communautés rurales : l'autre moteur économique du Québec. Québec, Québec : Groupe de travail sur la complémentarité rurale urbaine. Repéré à http://www.mamrot. gouv.qc.ca/pub/developpement_territorial/ruralite/grou pes_travail/etude_conferenceboard.pdf

${ }^{18}$ Canada 2020, op. cit.

${ }^{19}$ Mertins-Kirkwood, H. (2014). La mobilité de la maind'œuvre au Canada : problèmes et recomman-dations ayant trait aux politiques, rapport de recherche. Ottawa, Ontario : Congrès du travail du Canada. Repéré à http://congresdutravail.ca/sites/default/files/ media/labourmobility-rpt-hmk-2014-10-14-fr.pdf ${ }^{20}$ Grenier, A. (2012). Le marché du travail au Québec : horizon 2020. Communication présentée lors du congrès annuel de l'ACRGTQ, Québec.

${ }^{21}$ Mallett, T. (2013). Résultats du sondage de juillet 2013 sur les perspectives des PME. Baromètre des affaires. Ottawa, Ontario : Fédération canadienne de l'entreprise indépendante.

${ }^{22}$ Noreau, J. (2013). Main-d'œuvre au Québec : pénurie? rareté? Comment s'y retrouver?, Perspective (Desjardins Études économiques), 23.

${ }^{23}$ Desjardins, S. (2012). Cédants et repreneurs sous pression, Le Mentor, 5(3), 3.

${ }^{24}$ Raymond Chabot Grant Thornton. (2013). La relève entrepreneuriale : un enjeu de taille pour l'avenir économique du Québec. Repéré à http:// www.rcgt.com/wp-content/uploads/2013/11/Analyseinterne-RCGT-sondage-CROP-transfert-novembre2013-final.pdf

${ }^{25}$ Larochelle-Côté, S. et Uppal, S. (2011). Le bien-être financier des travailleurs autonomes. L'emploi et le revenu en perspective, 24(4). Repéré à http://www5.statcan.gc.ca/access_acces/alternative_alte rnatif.action?1=fra\&loc=../article/11535-fra.pdf

${ }^{26}$ Dostie, B. (2011). Réallocation de la main-d'œuvre et productivité au Canada, au Québec et en Ontario. Montréal, Québec : Centre sur la productivité et la prospérité, HEC Montréal. Repéré à http://cpp.hec.ca/cms/assets/documents/recherches_pub liees/CH-2010-01-rapport_fev.pdf

${ }^{27}$ Données recueillies lors d'une conférence prononcée par M. Benoit Dostie le 30 septembre 2015.

${ }^{28}$ Deslauriers, J., Gagné, R. et Paré, J. (2014). Croissance de la productivité au Québec : une perspective régionale. Montréal, Québec : Centre sur la productivité et la prospérité, HEC Montréal. Repéré à http://cpp.hec.ca/wp-content/uploads/2014/09/pp_ 2014_03.pdf

\section{NOTES}

${ }^{a}$ Le taux de fécondité, considérablement supérieur en région pendant de très nombreuses années, s'est lentement rapproché de celui des grands centres, pour se situer autour de 1,6\%, alors que le seuil pour assurer le renouvellement naturel de la population est supérieur à $2 \%$.

${ }^{b}$ Les principaux facteurs constatés qui influencent la diminution de la mobilité des jeunes adultes ruraux sont l'amélioration de l'offre de formations postsecondaires en région, la facilité d'accès à l'emploi et à la propriété, ainsi que l'amélioration des technologies de la communication. ${ }^{\mathrm{c}} \mathrm{La}$ ville de Montréal demeure plus que jamais la plaque tournante de la mobilité interrégionale, avec $48 \%$ des déplacements y étant rattachés à titre de point de départ ou de point d'arrivée. Cependant, bien que la métropole soit toujours en déficit dans ses échanges interrégionaux, son bilan migratoire s'améliore constamment depuis la première moitié des années 2000, et ce, particulièrement par rapport à ses régions périphériques que sont la Montérégie, Laval, les Laurentides et Lanaudière. Avec un taux d'accroissement de plus de $12,4 \%$, soit près de $4 \%$ supérieur à la moyenne québécoise, et avec une capacité accrue de rétention de sa population, Montréal réussit à maintenir, voire à améliorer son bassin de population active. dPour une cohorte donnée, il s'agit du nombre de ménages acheteurs sur le nombre de ménages au total.

${ }^{\text {eEntre }} 2001$ et 2006, le taux de propriétés des immigrants est passé de $68 \%$ à $72 \%$, soit une progression de $4 \%$, en comparaison à une progression de $2 \%$, pour un taux total de $75 \%$ du côté des Canadiens de souche.

${ }^{\mathrm{f}}$ Nombre de nouveaux arrivants par tranche de 100 personnes.

${ }^{\mathrm{g}}$ Les emplois de qualité élevée ont comme caractéristique d'être rémunérés $15,00 \$$ de l'heure ou plus (2002). Ils sont de qualification aussi élevée, c'est-à-dire technique, professionnelle et de gestion (ISQ, 2015).

helon certaines études (Riverain, 2005), les jeunes des régions ressources sont moins tournés vers l'entrepreneuriat que ceux des régions centrales. Par ailleurs, les résultats obtenus quant aux intentions d'entreprendre dans cette catégorie de population ne sont guère plus reluisants, bien que le récent sondage de 2014 de la Fondation de l'entrepreneurship paraisse indiquer une amélioration. Le retard des régions dans le domaine de l'entrepreneuriat semble également confirmé par l'ISQ dans ses statistiques sur la propension à entreprendre. 